\title{
Similarities and Differences Between Mild Cognitive Impairment and Alzheimer's Disease
}

\author{
Mark A. Lovell* \\ Sanders-Brown Center on Aging and Alzheimer's Disease Center, Department of Chemistry, University of \\ Kentucky, Lexington, Kentucky, USA
}

\section{INTRODUCTION}

This special issue is dedicated to discussion of similarities and differences between mild cognitive impairment (MCI) and Alzheimer's disease (AD). Amnestic MCI is thought to be a transition between normal aging and early dementia and may represent the best opportunity for pharmacologic interventions. Data suggest that conversion from MCI to dementia occurs at a rate of $10 \%$ to $15 \%$ per year [1] with $\sim 80 \%$ conversion by the sixth year of follow-up; although $\sim 5 \%$ of MCI subjects remain stable or convert back to normal $[2,3]$. With the description of MCI as a transition between normal aging and dementia, recent emphasis has focused on early disease detection and elucidation of pathogenic mechanisms that underlie progression to $\mathrm{AD}$ with the hope of early treatment to stop or slow progression of the disease. Over the past few years multiple studies have examined pathogenic changes in MCI brain and have demonstrated changes that are comparable to changes observed in late stage AD.

\footnotetext{
*Correspondence to: Mark A. Lovell, Sanders-Brown Center on Aging and Alzheimer's Disease Center, Department of Chemistry, University of Kentucky, 135 Sanders-Brown Building, 800 S. Limestone Street, Lexington, Kentucky 40536, USA. Tel.: +1 859257 1412 x 251; Fax: +1 859323 2866; Email: malove2@email.uky.edu.
}

The goal of this issue was to assemble a series of articles in one place that compare and contrast various aspects of MCI/AD including clinical parameters, potential genetic components, imaging (MRI, PET/SPECT), and neuropathological alterations that occur in the progression from MCI to AD. The issue presents both scholarly reviews of current literature and reports of new research findings and provides a diverse view of the differences and similarities between MCI and AD. The comparison between MCI and AD may provide insight into the pathogenic features of $\mathrm{AD}$ that occur early in the disease and thus identify potential therapeutic targets aimed at slowing progression or perhaps preventing the onset of $\mathrm{AD}$.

\section{REFERENCES}

[1] Petersen RC, Morris JC (2003) Clinical features. In: Mild Cognitive Impairment, Petersen RC, ed., Oxford University, New York, pp. 15-39.

[2] Bennett DA, Wilson RS, Schneider JA, Evans DA, Beckett LA, Aggarwal NT, Barnes LL, Fox JH, Bach J (2002) Natural history of mild cognitive impairment in older persons. Neurology 59, 198-205.

[3] DeCarli C (2003) Mild cognitive impairment: prevalence, prognosis, aetiology, and treatment. Lancet Neurol 2, 15-21. 